\title{
Paradigms of shared knowledge
}

\author{
Gerry Stahl • Friedrich Hesse
}

Published online: 15 September 2009

(C) International Society of the Learning Sciences, Inc.; Springer Science + Business Media, LLC 2009

\section{Multiple paradigms for analyzing shared knowledge in collaboration}

Collaborative learning is all about sharing knowledge. Without a shared base of knowledge (common ground), discourse itself is impossible, let alone collaborative interaction. Collaborators must share a means of communication (language), a joint focus (object-orientation), and a compatible orientation (perspective). In addition to being dependent upon the presence of existing shared knowledge, successful collaboration or collaborative learning involves the construction of new knowledge, created jointly and thereby shared by the participants. Knowledge can take many forms, not necessarily rational, propositional, explicit, factual knowledge. There is tacit and explicit knowledge, focal and background, propositional and procedural, personal and institutional, individual and group.

Studies of CSCL each tend to focus on a certain form of knowledge and assume a certain way of sharing this knowledge. These choices depend upon the theoretical position implicitly or explicitly adopted by the study. You may find it interesting to figure out which paradigm of shared knowledge corresponds to each of the articles in this issue.

The topic of paradigms of shared knowledge may seem abstrusely theoretical and remote from the practical concerns of CSCL. However, it strongly affects whether a given educational intervention-incorporating pedagogical resources, computer technologies, scripting, grouping of students - will foster effective collaborative learning. In fact, it may affect this even more when the teachers, researchers, or other CSCL designers are not explicitly aware of their assumptions about shared knowledge.

The four articles of this issue, which deal with computer support for shared knowledge, are all focused on the practical design of technologies to support collaborative learning: wikis, virtual reality, PowerPoint, and group formation software. They each presume a

G. Stahl $(\bowtie)$

Drexel University, Philadelphia, PA, USA

e-mail: Gerry.Stahl@drexel.edu

F. Hesse

Knowledge Media Research Center, Tubingen, Germany

e-mail: f.hesse@iwm-kmrc.de 
different paradigm of shared knowledge. The following paragraphs define four paradigms spanning a range:

The paradigm of sharing individual mental representations

Perhaps the most commonsensical view of shared knowledge in a small group is that the individual members of the group each possess the same knowledge. This can be elaborated theoretically by hypothesizing that each member has mental representations that are sufficiently similar to specific mental representations of each of the other members. The classic analysis of grounding (Clark and Brennan 1991) that is often cited in CSCL research describes how two typical collaborators might establish shared knowledge by externalizing their ideas and explicitly comparing the propositional expressions of their mental representations. This paradigm assumes that individuals possess well-formed opinions and can unproblematically express them. Sharing is here taken to be a matter of transferring and comparing ideas in ways that typically do not change the ideas.

The paradigm of sharing an object

A quite different view conceives of shared knowledge as a natural consequence of a group being collaboratively involved with the object of their work together. They are all oriented in common toward the same object (an artifact, a problem, a goal) and thereby come to share knowledge of that object, in particular the knowledge about that object that arises from their work with it. The sharing of knowledge about a common object does not need special coordination, once the object is truly shared. A recent ijCSCL article (Çakır et al. 2009) described an example of how students in an online group worked to define and share multiple realizations of a mathematical object; once they could all "see" the same object, the construction of new shared knowledge (such as the formulation of an algebraic expression to solve their problem) proceeded quickly. Another recent article (Dohn 2009) discussed how the affordances of an object must be enacted; in the collaborative case, this is accomplished interactively as the group comes to know and share the object. In both analyses, the shared knowledge is new knowledge for all the participants, arising out of their interactions with each other, with the shared object, and with other resources for communication and understanding, including available computer supports.

The paradigm of sharing a situation

If we broaden the notion that shared knowledge comes from a joint focus on an object of collaboration, we come to the idea that a group can share knowledge by being situated in a common context - a joint problem space (Roschelle and Teasley 1995) or an indexical ground of reference (Hanks 1992). The situation includes the shared object, but it also includes other resources and constraints, such as the affordances of a CSCL environment. Above all, it includes the past discourse of the group, which has created a complex network of shared concepts, interactions and experiences. Duranti and Goodwin (1992) emphasize that the situation and the discourse "stand in a mutually constitutive relationship to each other, with talk, and the interpretive work it generates, shaping context as much as context shapes talk" (p. 31). According to this paradigm, the engagement in collaborative discourse can automatically generate shared knowledge as an ongoing process. 
The paradigm of sharing a community

The social sciences generally take an even broader view. They argue that the shared knowledge that makes life together possible comes from belonging to the same communities, cultures, and societies. It is the understanding of the same historically accumulated knowledge, values, perspectives, artifacts, and ways of life-largely encapsulated in language - that makes communication possible. In proposing that we broaden our thinking about computer support to include complex technological infrastructures, Jones et al. (2006) tried to show how the institutional macro-level could be related to the mezzo-level of collaboration and even to the micro-level of fine-grained interaction analysis.

The existence of multiple effective paradigms for understanding something like shared knowledge is not necessarily problematic. It may be possible to select the most appropriate paradigm for any given study. However, it does raise the question of how the paradigms might fit together - a topic for another time. Now we turn to four concrete proposals for supporting shared knowledge.

\section{Intersubjectivity in collaborative learning}

In the first article of this issue, Johann Larusson and Richard Alterman claim that wiki technology is particularly suited for enabling students to create an online intersubjective space that supports their collaboration. The wiki's malleable and easy-to-use interface, which contributes to its broad applicability, is, however, in need of specific kinds of additional functionality for collaborative learning settings. The paper describes a Wiki Design Platform that provides a suite of awareness, navigation, communication, and analysis components and scaffolds.

Two case studies demonstrate how different selections of components from the suite can help create an online intersubjective space for quite different forms of collaboration in a college classroom. In the first case study, student teams collaborate on HCI design projects. In the second, the students co-blog in the wiki about their course readings.

One might think that the design projects foster shared knowledge through the joint focus on the artifact being designed, while the co-blogging supported individuals externalizing their individual understandings. In fact, the paradigm of shared knowledge in this article is more complex because the authors refer to their theory of intersubjectivity (Alterman 2007). For them, the intersubjective space is partially biological, but also social and cultural. In addition, it is related to the history of individual and group activities, and it provides a background for individuals to interpret each other's actions and (mental) motives. It is, therefore, not immediately clear which paradigm the presented concept of intersubjectivity falls into, or what form of shared knowledge is central to the suite of wiki tools or to the educational interventions in the case studies.

\section{Collaborative learning in a mixed-reality classroom}

The design of SMALLab, as presented by David Birchfield and Colleen MegowanRomanowicz, pivots on three principles:

- Direct face-to-face interaction among co-located participants within the computationally mediated space should be cultivated. 
- Thought and action should be distributed across multiple participants through an active, generative process that unfolds in real time.

- Immediate (spatial and temporal) consolidation of emergent conceptual models should follow the active learning process.

Each of these design principles supports shared knowledge. They provide the preconditions and mechanisms for knowledge to be shared among students in a classroom through their structured interaction.

In addition, the mixed-reality intervention involves the computer-supported projection of a virtual reality into the physical space of the classroom, interacting with the behaviors of students. This creates an environmental situation, embodying dramatic and interactive representations of otherwise abstract earth sciences concepts. The reported study demonstrates that situating groups of students in such a mixed-reality setting can be highly motivating even for at-risk students, leading to their construction of scientific shared knowledge.

Together, the design principles, the mixed-reality technology, and the whole-class activities may be considered to bring together the paradigms of individual, object-oriented, situated, and community knowledge sharing.

\section{Tools for presentation and critique in education}

Architectural education traditionally employs extensive use of apprenticeship modes of sharing knowledge (Schön 1983). In particular, design studios are scripted occasions for students to present designs and for professionals to publically reflect on them. Through careful conversation analysis of design studio sessions, Gustav Lymer, Jonas Ivarsson, and Oskar Lindwall investigate the effects of different technologies - such as paper posters, PowerPoint slideshows, and combinations thereof-for supporting presentation and critique in such sessions.

For knowledge to be shared, it is necessary that the participants can see the same thing in the same way. In the two previous articles, this was an important, but implicit principle. The wiki and the virtual reality were designed to create shared perceptual spaces, where salient objects could be seen by all. The previously referenced article, (Çakır et al. 2009), analyzed how students explicitly shared their ways of seeing in an online setting-much as (Goodwin 1994) did for face-to-face settings. Here, the authors tease apart the ways in which presentation technologies mediate the sharing of ways of seeing, an important constituent of sharing knowledge.

\section{Theory-driven group formation}

In the previous articles, the authors design an interaction space or educational setting into which teachers can place groups of students. The question then arises as to how to form student groups that will engage in optimal collaborative learning processes within the given spaces. In the final article of ijCSCL volume four, Seiji Isotani, Akiko Inaba, Mitsuru Ikeda, and Riichiro Mizoguchi propose the reverse procedure. They start from the individual students' educational needs and goals to then form group activities that are responsive to those needs. Of course, to expect classroom teachers to match each of their students' needs to appropriate CSCL theories, technologies and pedagogies - and then to 
form compatible groupings of students in selected activities - without adequate support is not feasible.

The paper, therefore, presents a framework or ontology of categories for analyzing, specifying, and coordinating student needs, theories, technologies, and best practices. By starting from the needs of individual students, the authors hypothesize that it will be possible to group together students who can support one another and to select personally tuned collaborative-learning activities that can help the members of a group to achieve their goals as individuals and as a group. It is important to keep in mind when reading this technical paper that it is not trying to automate group formation, but to support teachers in their role as facilitators of collaboration and orchestrators of knowledge sharing.

\section{References}

Alterman, R. (2007). Representation, interaction, and intersubjectivity. Cognitive Science, 31(5), 815-841.

Çakır, M. P. Zemel, A. \& Stahl, G. (2009). The joint organization of interaction within a multimodal CSCL medium. International Journal of Computer-Supported Collaborative Learning, 4(2), 115-149. doi:10.1007/s11412-009-9061-0.

Clark, H. \& Brennan, S. (1991). Grounding in communication. In L. Resnick, J. Levine \& S. Teasley (Eds.), Perspectives on socially-shared cognition (pp. 127-149). Washington: APA.

Dohn, N. B. (2009). Affordances revisited: articulating a Merleau-Pontian view. International Journal of Computer-Supported Collaborative Learning, 4(2), 151-170. doi:10.1007/s11412-009-9062-z.

Duranti, A. \& Goodwin, C. (eds). (1992). Rethinking context: Language as an interactive phenomenon. Cambridge: Cambridge University Press.

Goodwin, C. (1994). Professional vision. American Anthropologist, 96(3), 606-633.

Hanks, W. (1992). The indexical ground of deictic reference. In A. Duranti \& C. Goodwin (Eds.), Rethinking context: Language as an interactive phenomenon (pp. 43-76). Cambridge: Cambridge University Press.

Jones, C. Dirckinck-Holmfeld, L. \& Lindström, B. (2006). A relational, indirect, meso-level approach to CSCL design in the next decade. International Journal of Computer-Supported Collaborative Learning, l(1), 35-56. doi:10.1007/s11412-006-6841-7.

Roschelle, J. \& Teasley, S. (1995). The construction of shared knowledge in collaborative problem solving. In C. O'Malley (Ed.), Computer-supported collaborative learning (pp. 69-197). Berlin: Springer Verlag. Schön, D. A. (1983). The reflective practitioner: How professionals think in action. New York: Basic Books. 